\title{
Projective Limits of Vector Measures
}

\author{
Fidel J. Fernández and P. Jiménez Guerra
}

\begin{abstract}
A necessary and sufficient condition for the existence of the projective limit of measures with values in a locally convex space is given. A similar theorem for measures with values in different locally convex spaces (under certain conditions) is given too (in this case, the projective limit is valued in the projective limit of these spaces). Finally, a result about the projective limit of vector measures is stated.
\end{abstract}

\section{INTRODUCTION AND NOTATION}

In [26] L. Schwartz has proved the Prokhorov's theorem about the existence of the projective limit of a projective system of finite (scalar) Radon measures (of type $(\mathscr{K})$ ) on Hausdorff topological spaces. This result has been extended in [16] for arbitrary (scalar) Radon measures of type $(\mathscr{H})$ on topological spaces.

As it is well known the Prokhorov's theorem has a very important role in the study of cylindrical measures and in general in probability theory.

The main object of this paper is to prove a Prokhorov's type theorem for vector Radon measures. This has been made here for Radon measures of type $(\not /)$ on an arbitrary topological space $E$ with values in a complete locally convex Hausdorff space $X$. Of special interest are the following particular cases: (1) $E$ is a Hausdorff topological space and $\mathscr{H}$ is the class of all compact subsets of $E$, and (2) $X$ is a Banach space.

In the last section we give the relation between the projective limit of a system of product measures $\left(\mu_{i} \otimes \nu_{i}\right)_{i \epsilon}$ and the tensor product of the limits of the systems $\left(\mu_{i}\right)_{i \epsilon I}$ and $\left(\nu_{i}\right)_{i \epsilon}$. This result remains valid in general without any assumption about the regularity of the measures. A theorem of this type for scalar Radon measures has been proved in [12].

1980 Mathematics Subject Classification (1985 revision): 28B05. Editorial de la Universidad Complutense. Madrid, 1990. 
Let $X$ be a complete locally convex Hausdorff space whose topology is defined by a saturated family $\mathscr{P}$ of seminorms, and denote by $E, \mathscr{G}, \mathscr{F}$ and $\mathscr{B}$ a topological space and the classes of its open, closed and Borel subsets respectively.

If $\mu: \mathscr{B} \rightarrow X$ is a ( $\sigma$-additive) vector measure, and $p \in \mathscr{P}$, the $p$ semivariation of $\mu$ will be as usual the mapping $\|\mu\|_{p}: \mathscr{B} \longrightarrow \mathbb{R}+\cup\{+\infty\}$ defined by

$$
\|\mu\|_{p}(B)=\sup p\left(\sum_{j=1}^{n} t_{j} \mu\left(A_{j}\right)\right) \quad(B \in \mathscr{D}) \quad,
$$

where the supremum is taken over all partitions of $B$ into a finite number of disjoint sets $\left\{A_{j}\right\}_{j=1}^{n} \subset \mathscr{B}$ and all finite collections of elements $\left\{t_{j}\right\}_{j=1}^{n} \subset \mathbb{R}$ with $\left|t_{j}\right| \leq 1$ for every $j=1, \ldots, n$. It is easily proved that

$$
\|\mu\|_{p}(B)=\sup \left(\sum_{j=1}^{n}\left|x^{\prime} \mu\left(A_{j}\right)\right|\right) \quad(B \in \mathscr{D}) \quad,
$$

where the supremum is taken over all finite partitions $\left\{A_{j}\right\}_{j}{ }_{1}{ }_{1} \subset \mathscr{B}$ of $B$ and all $x^{\prime} \in X^{\prime}$ such that $\left|x^{\prime}(y)\right| \leq p(y)$ for every $y \in X$, where $X^{\prime}$ denotes the dual space of $X$; and

$$
\|\mu\|_{p}(B) \leq 2 \sup \{p(\mu(A)): A \in \mathscr{B}, A \subset B\}
$$

for every borel subset $B \in \mathscr{B}$.

\section{EXISTENCE THEOREMS FOR PROJECTIVE LIMITS OF VECTOR MEASURES}

Definition 1. A borel subset $B \in \mathscr{B}$ is said to be $\mu$-compact if for every open cover $\left\{G_{i}\right\}_{i \epsilon}$ of $B$, every seminorm $p \in \mathscr{P}$ and $\epsilon>0$, there exisis a finite subset $J \subset I$ such that

$$
\|\mu\|_{p}\left(B-\bigcup_{i \in J} G_{i}\right) \leq \epsilon
$$

Definition 2. Let $\mathscr{Y}$ be a family of closed subsets of $E$. We say that $\mu$ is a Radon measure of type ( $\mathscr{A})$ if the following statements hold :

\subsection{Every $H \in \mathscr{Z}$ is $\mu$-compact.}

2.2. For every $B \in \mathscr{B}, p \in \mathscr{P}$ and $\epsilon>0$, there exists $H \in \mathscr{C}$ such that $H \subset B$ and $\|\mu\|_{p}(B-H) \leq \epsilon$.

If $\mu$ is a Radon measure of type $(\mathscr{H})$ then it is easily proved that every Borel subset of $E$ is $\mu$-compact. 
Definition 3. Let $E$ be a projective limit of the projective system ( $\left.E_{i,}, \Pi_{i j}\right)_{i, j \in l}$ of topological spaces and denote by $\Pi_{i}$ the corresponding projection from $E$ into $E_{1}(i \in I)$. If for every $i \in I, \mu_{i}: \mathscr{D}_{i} \rightarrow X$ is a Radon measure of type $\left(\mathscr{\mathscr { X }}_{i}\right)\left(\mathscr{B}_{i}\right.$ denotes the Borel family of $E_{i}$; and $\mathscr{K}_{i}$, a family of closed subsets of $E_{i}$, which is closed under finite unions), we say that $\left(\mu_{i}\right)_{i \in t}$ is a projective system of Radon measures of type $\left(\mathscr{W}_{i}\right)$, if $\Pi_{i j}\left(\mu_{j}\right)=\mu_{i}$ (i.e. $\mu_{i}\left(B_{i}\right)=\mu_{j}\left(\Pi_{i j}^{-}\left(B_{i}\right)\right\}$ for all $\left.B_{i} \in \mathscr{B}_{i}\right)$ for every $i, j \in I$ with $i \leq j$; and a Radon measure of type (\&) $\mu: S B \rightarrow X$ is said to be the projective limit of the measures $\left(\mu_{i}\right)_{i \in \text { I }}$ (it is immediately proved that if the projective limit measure exists then it is unique) when $\Pi_{i}(\mu)=\mu_{i}$ holds for every $i \in I$ (i.e. $\mu_{i}\left(B_{i}\right)=$ $=\mu\left(\Pi_{i}^{-I}\left(B_{i}\right)\right)$ for every $B_{i} \in \mathscr{D}_{i}$ and $\left.i \in I\right)$.

Let us introduce the following conditions:

3.1. $\mathscr{\mathscr { O }}$ is closed under finite unions, $H \cap F \epsilon \mathscr{H}$ for every $H \epsilon \mathscr{H}$ and $F \epsilon \mathscr{G}, \overline{\Pi_{i}(H)} \epsilon \mathscr{K} i$ for every $H \epsilon \mathscr{H}$ and $i \epsilon I$, and for every $H \epsilon \mathscr{H}$ there exists $i_{H} \in I$ such that $\Pi_{i}(H) \in \mathscr{B}_{i}$ for every $i \geq i_{H}$.

3.2. For every $i \in I$ and $p \in \mathscr{P}$, there exists a non negative and finite measure $\nu_{i}^{p}: S_{i} \longrightarrow \mathbb{R}^{+}$such that

3.2.1. $\Pi_{i j}\left(\nu_{j}^{p}\right)=\nu_{i}^{p}$ for every $p \epsilon \mathscr{D}$ and $i, j \in I$ with $i \leq j$.

3.2.2. $\nu_{i}^{p}\left(B_{i}\right)=\inf \left\{\nu_{i}^{p}\left(G_{i}\right): B_{i} \subset G_{i} \in \mathscr{G}_{i}\right\}$, for every $i \in I, p \in \mathscr{P}$ and $B_{i} \in S_{i}$.

3.2.3. For every $\epsilon>0$ and $p \epsilon \in$ there exists $i_{o} \epsilon I$ and $\eta>0$ such that $\left\|\mu_{i}\right\|_{p}\left(B_{i}\right) \leq \epsilon$ for all $i \geq i_{o}$ and $B_{i} \in \mathscr{B}_{i}$ with $\nu_{i}^{p}\left(B_{i}\right) \leq \eta$.

Lemma 4. If the Radon measure of type $(\mathscr{C}) \mu: \mathscr{B} \rightarrow X$ is the projective limit of a projective system $\left(\mu_{i}\right)_{i \in I}$ of Radon measures of type $\left(\mathscr{C}_{i}\right)$ and conditions 3.1 and 3.2 are verified, then

$$
\mu(H)=\lim _{j \geq i_{H}} \mu_{j}\left(\Pi_{j}(H)\right)
$$

for every $H \epsilon \notin$ Moreover, $\mu$ is of bounded semivariation if and only if the semivariations of the measures $\mu_{i}$ (i $\left.\in I\right)$ are uniformly bounded.

Proof. It follows from conditions 3.1 and 3.2 that for every $H \epsilon \mathscr{H}$, $\left(\mu_{i}\left(\Pi_{i}(H)\right)\right)_{i \geq i_{H f}}$ is a Cauchy net in $X$.

Let us set

$$
\lambda(H)=\lim _{i \geq i_{i f}} \mu_{i}\left(\Pi_{i}(H)\right)
$$


(If the semivariations $\left(\left\|\mu_{i}\right\|_{p}\right)_{i \epsilon I}$ are uniformly bounded (for every $\left.p \epsilon 9\right)$ ), then $\left(\mu_{i}\left(\Pi_{i}(H)\right)\right)_{i \geq i_{H}}$ is a bounded Cauchy net in $X$ and so the limit [4.2] exists also assuming only that the space $X$ is quasi-complete.)

Let $H \epsilon \mathscr{\mathscr { C }}, p \in \mathscr{P}$ and $\epsilon>0$. Then

$$
H=\bigcap_{\substack{i \in l \\ i \geq i_{H}}} \Pi_{i}^{-1} \Pi_{i}(H)=\bigcap_{\substack{i \in l \\ i \geq i_{H}}} \Pi_{i}^{-1}\left(\overline{\Pi_{i}(H)}\right),
$$

and there exists $i_{1}, \ldots, i_{r} \in I$ such that

$$
\|\mu\|_{p}\left(\left(\Pi_{i_{H}}^{-1}\left(\overline{\Pi_{i_{H}}(H)}\right)-H\right) \cap\left(\bigcap_{h=1}^{r} \Pi_{i_{h}}^{-1}\left(\overline{\Pi_{i_{h}}(H)}\right)\right)\right) \leq \epsilon .
$$

Therefore, if $i_{o} \in I$ is such that $i_{H} \leq i_{o}$ and $i_{h} \leq i_{o}$ for $h=1, \ldots, r$, then

$$
\|\mu\|_{p}\left(\Pi_{i}^{-1} \Pi_{i}(H)-H\right) \leq \epsilon
$$

for every $i \in I$ with $i \geq i_{s}$, and

$$
\begin{aligned}
& p\left(\mu_{i}\left(\Pi_{j}(H)\right)-\mu(H)\right)=p\left(\mu\left(\Pi_{i}^{-1} \Pi_{i}(H)\right)-\mu(H)\right)= \\
& =p\left(\mu\left(\Pi_{i}^{-1} \Pi_{i}(H)-H\right)\right) \leq\|\mu\|_{p}\left(\Pi_{i}^{-1} \Pi_{i}(H)-H\right) \leq \epsilon
\end{aligned}
$$

for every $i \epsilon I$ with $i_{o} \leq i$. So, $p(\lambda(H)-\mu(H)) \leq \epsilon$;

from where it follows immediately that $\mu(H)=\lambda(H)$.

Moreover, if $\mu$ is of bounded semivariarion then the measures $\mu_{i}(i \in I)$ are of uniformly bounded semivariation since

$$
\left\|\mu_{i}\right\|_{p}\left(B_{i}\right) \leq\|\mu\|_{p}\left(\Pi_{i}^{-1}\left(B_{i}\right)\right),
$$

for all $p \in \mathscr{P}$ and $B_{i} \epsilon \mathscr{B}_{i}$. Conversely, if for every $p \epsilon \mathscr{P}$ there exists $K>0$ such that $\left\|\mu_{i}\right\|_{p}\left(E_{i}\right) \leq K$ for every $i \epsilon I$, then for every $\epsilon>0$ and $B \epsilon \mathscr{B}$, there exists $H \epsilon \mathscr{\mathbb { Z }}$ such that $H \subset B$ and $\|\mu\|_{p}(B-H) \leq \epsilon$; therefore ,

$$
\begin{gathered}
p(\mu(B)) \leq p(\mu(B-H))+p(\mu(H)) \leq\|\mu\|_{p}(B-H)+ \\
+p(\lambda(H)) \leq \epsilon+\sup _{\substack{i \in I \\
i \geq i_{H}}}\left\|\mu_{i}\right\|_{p}\left(E_{i}\right) \leq \epsilon+K
\end{gathered}
$$

and $\|\mu\|_{p}(E) \leq 2 \cdot \sup \{p(\mu(B)): B \epsilon \mathscr{B}\} \leq 2 K<+\infty$.

Theorem 5. Let $\left(\mu_{\nu_{i \in}}\right.$ be a projective system of Radon measures of type $\left(\mathscr{Y}_{i}\right)$, and assume that conditions 3.1 and 3.2 are satisfied. Then the projective 
limit $\mu$ of the system $\left(\mu_{i}\right)_{i t}$ exists and $\mu: S-X$ is a Radon measure of type (\&), if and only if the following statements hold:

5.1. For every $p \epsilon \mathscr{P}, \epsilon>0, H \epsilon \mathscr{H}$ and open cover $\left\{G_{j}\right\}_{j \epsilon} J$ of $H$, there exists a finite subset $J^{\prime} \subset J$ and $i_{o} \in I$ such that $i_{o} \geq i_{H_{l}}$, with $H_{1}=H-\bigcup_{j \in J^{\prime}} G_{j}$, and

$$
\left\|\mu_{i}\right\|_{p}\left(\Pi_{i}\left(H-\bigcup_{j \epsilon J^{*}} G_{j}\right)\right)<\epsilon
$$

for every $i \geq i_{\phi}$.

5.2. For every $p \in \mathscr{P}, \epsilon>0$, iє I and $H_{i} \in \mathscr{H}_{i}$, there exists $H \in \mathscr{Z}$ such that $H \subset \Pi_{i}^{-1}\left(H_{i}\right)$ and

$$
\left\|\mu_{i},\right\|_{p}\left(\Pi_{i i}^{-I}\left(H_{i}\right)-\Pi_{i,}(H)\right) \leq \epsilon,
$$

for every $i^{\prime} \in I$ with $i^{\prime} \geq i$ and $i^{\prime \prime} \geq i_{H}$.

Proof. The condition is necessary. Consider $p \epsilon \mathscr{P}, \epsilon>0$ and $H \epsilon \mathscr{H}$. If $\left\{G_{j}\right\}_{j \epsilon J}$ is an open cover of $H$, there exists a finite subset $J^{\prime} \subset J$ such that

$$
|| \mu \|_{p}\left(H-\bigcup_{j \in J} G_{j}\right) \leq \epsilon / 2 .
$$

Set $H_{1}=H-\bigcup_{j \in J^{*}} G_{j} \in \mathscr{K} ;$ then as we have seen in the proof of Lemma 4, there exists $i_{o} \in I$ such that $i_{o} \geqq i_{H_{1}}$ and

$$
\|\mu\|_{p}\left(\Pi_{i}^{-1} \Pi_{i}\left(H_{1}\right)-H_{1}\right) \leq \epsilon / 2
$$

for all $i \epsilon I$ with $i \geq i_{o}$. Therefore, we get

$$
\begin{gathered}
\left\|\mu_{i}\right\|_{p}\left(\Pi_{i}\left(H-\bigcup_{j \epsilon J^{\prime}} G_{j}\right)\right) \leq|| \mu \|_{p}\left(\Pi_{i}^{-1} \Pi_{i}\left(H_{l}\right)\right) \leq \\
\leq\|\mu\|_{p}\left(\Pi_{i}^{-1} \Pi_{i}\left(H_{1}\right)-H_{1}\right)+\|\mu\|_{p}\left(H_{1}\right) \leq \epsilon,
\end{gathered}
$$

for all $i \geq i_{o}(i \in I)$, and 5.1 holds.

Moreover, for $i \epsilon I, H_{i} \epsilon \mathscr{H}_{i}, p \in \mathscr{P}$ and $\epsilon>0$, there exists $H \epsilon \mathscr{W}$ such that $H \subset \Pi_{i}^{-1}\left(H_{i}\right)$ and

$$
\|\mu\|_{p}\left(\Pi_{i}^{-1}\left(H_{i}\right)-H\right) \leq \epsilon
$$

Therefore we get, if $j \geq i$ and $j \geq i_{H}(j \in I)$,

$$
\begin{gathered}
|| \mu_{j}\left\|_{p}\left(\Pi_{i j}^{-1}\left(H_{i}\right)-\Pi_{j}(H)\right) \leq\right\| \mu \|_{p}\left(\Pi_{j}^{-1}\left(\Pi_{i j}^{-1}\left(H_{i}\right)-\Pi_{j}(H)\right)\right)= \\
=\|\mu\|_{p}\left(\Pi_{i}^{-1}\left(H_{i}\right)-\Pi_{j}^{-1} \Pi_{j}(H)\right) \leq\|\mu \mid\|_{p}\left(\Pi_{i}^{-1}\left(H_{i}\right)-H\right) \leq \epsilon, \text { and } 5.2 \text { holds. }
\end{gathered}
$$


The condition is sufficient. Let $\lambda$ be defined as in [4.2]; then we nave:

i) If $H_{1}, H_{2} \in \dot{y}$ satisfy $H_{1} \cap H_{2}=\emptyset$, then

$$
\lambda\left(H_{1} \cup H_{2}\right)=\lambda\left(H_{1}\right)+\lambda\left(H_{2}\right) .
$$

In fact, $H_{1} \subset E-H_{2}=\bigcup_{i \in I}\left(E-\Pi_{i}^{-1}\left(\overline{\Pi_{i}\left(H_{2}\right)}\right)\right)$, and it follows from 5.1 that for every seminorm $p \epsilon \mathscr{P}^{i \epsilon}$ and $\epsilon>0$ there exists $i_{o} \epsilon I$ such that

$\left\|\mu_{j}\right\|_{p}\left(\Pi_{j}(B)\right) \leq \epsilon$ for every $B \epsilon \mathscr{B}$ and $j \in I$ such that $j \geq i_{o}$, $B \subset H_{1} \cap \Pi_{j}^{-1}\left(\overline{\Pi_{j}\left(H_{2}\right)}\right)$ and $\Pi_{j}(B) \in \mathscr{B}_{j}$. Therefore,

$p\left(\lambda\left(H_{1}\right)+\lambda\left(H_{2}\right)-\lambda\left(H_{1} \cup H_{2}\right)\right)=$

$=p\left(\lim _{\substack{i \in I \\ i \geq i_{H_{1}}, i_{H_{2}}, i_{H_{1}} \cup H_{2}}}\left(\mu_{i}\left(\Pi_{i}\left(H_{1}\right)\right)+\mu_{i}\left(\Pi_{i}\left(H_{2}\right)\right)-\mu_{i}\left(\Pi_{i}\left(H_{1} \cup H_{2}\right)\right)\right)=\right.$

$=\lim _{\substack{i \in I \\ i \geq i_{H_{1}}, i_{H_{2}}, i_{o}}} p\left(\mu_{i}\left(\Pi_{i}\left(H_{1}\right) \cap \Pi_{i}\left(H_{2}\right)\right)\right) \leq \sup _{\mathbb{R} i_{i_{0}, i_{H_{1}}, i_{H_{2}}}}\left\|\mu_{i}\right\|_{p}\left(\Pi_{i}\left(H_{1}\right) \cap \Pi_{i}\left(H_{2}\right)\right)=$

$=\sup _{i \geq i_{o}, i_{H_{1}}, i_{H_{2}}}\left\|\mu_{i}\right\|_{p}\left(\Pi_{i}\left(H_{1} \cap \Pi_{i}^{-1} \Pi_{i}\left(H_{2}\right)\right)\right) \leq \epsilon$,

and the equality [5.1] holds.

ii) For every $H \epsilon \mathscr{H}, p \epsilon \mathscr{P}$ and $\epsilon>0$, there exists $G \epsilon \mathscr{G}$ such that $H \subset G$ and

$$
p\left(\lambda\left(H^{\prime}\right)-\lambda\left(H^{\prime \prime}\right)-\lambda(H)\right) \leq \epsilon
$$

if $H^{\prime}, H^{\prime \prime} \in \mathscr{H}, H \subset H^{\prime}$ and $H^{\prime}-G \subset H^{\prime \prime} \subset H^{\prime}-H$.

To prove this, let us remark first that for every $H \epsilon \& / f, p \epsilon \rightarrow$ and $\epsilon>0$, there exists $i_{o} \geq i_{H}\left(i_{o} \in I\right)$ such that if $k \geq j \geq i_{o}(k, j \in I)$, then

$$
\left\|\mu_{k}\right\|_{p}\left(\Pi_{j k}^{-1}\left(\Pi_{j}(H)\right)-\Pi_{k}(H)\right) \leq \epsilon / 2 .
$$

Moreover, there exists $\eta>0$ and $i_{1} \epsilon I$ such that $\left\|\mu_{j} \mid\right\|_{p}\left(B_{i}\right) \leq \epsilon / 2$ for every $i \geq i_{1}(i \in I)$ and $B_{i} \in \mathscr{B}_{i}$ with $\nu_{i}^{p}\left(B_{i}\right) \leq \eta$.

Let us consider $j \geq i_{o}, i_{1}$. Since $i_{o} \geq i_{H}, \Pi_{j}(H) \in \mathscr{B}_{j}$ and then

$$
\nu_{j}^{p}\left(\Pi_{j}(H)\right)=\inf \left\{\nu_{j}^{p}(G): \Pi_{j}(H) \subset G \in \mathscr{G}_{j}\right\}
$$

and there exists $G_{j} \in \mathscr{G}_{j}$ such that $\Pi_{j}(H) \subset G_{j}$ and $\nu_{j}^{p}\left(G_{i}-\Pi_{j}(H)\right) \leq \eta$.

So we have

$\nu_{i}^{p}\left(\Pi_{j i}^{-1}\left(G_{j}\right)-\Pi_{j i}^{-1}\left(\Pi_{j}(H)\right)\right)=\nu_{j}^{p}\left(G_{j}-\mathrm{II}_{j}(H)\right) \leq \eta$, 
and

||$\mu_{i}||_{p}\left(\Pi_{j l}^{-1}\left(G_{j}\right)-\Pi_{i}(H)\right) \leq$

$\leq\left\|\mu_{j}\right\|_{p}\left(\Pi_{j i}^{-1}\left(G_{j}\right)-\Pi_{j i}^{-1} \Pi_{i}(H)\right)+\left\|\mu_{i}\right\|_{p}\left(\Pi_{j i}^{-1} \Pi_{i}(H)-\Pi_{i}(H)\right) \leq$

$\leq \epsilon / 2+\epsilon / 2=\epsilon$,

if $i \geq j \quad(i \in \Lambda)$.

Set $G=\Pi_{j}^{-1}\left(G_{j}\right)$; then $H \subset G$ and

$\Pi_{i}\left(H^{\prime}\right)-\Pi_{i}\left(H^{\prime \prime}\right) \subset \Pi_{j i}^{-1}\left(G_{j}\right)$ if $H^{\prime}, H^{\prime \prime} \in \mathscr{H}, H \subset H^{\prime}$,

$H^{\prime}-G \subset H^{\prime \prime} \subset H^{\prime}-H$ and $i \geq j$. Therefore,

$p\left(\lambda\left(H^{\prime}\right)-\lambda\left(H^{\prime}\right)-\lambda(H)\right)=p\left(\lambda\left(H^{\prime}\right)-\lambda\left(H^{\prime \prime} \cup H\right)\right)=$

$=p\left(\lim _{i \geq j, i_{H^{*}}, i_{H \cup H^{m}}}\left(\mu_{i}\left(\Pi_{i}\left(H^{\prime}\right)\right)-\mu_{i}\left(\Pi_{i}\left(H^{\prime \prime} \cup H\right)\right)\right)\right)=$

$=\lim _{i \geq_{j, i_{H}, i_{H} \cup H^{r}}} p\left(\mu_{i}\left(\Pi_{i}\left(H^{\prime}\right)-\Pi_{i}\left(H^{\prime \prime} \cup H\right)\right)\right) \leq$

$\leq \sup _{i \geq j, i_{H^{*}, i_{H} \cup H^{\prime \prime}}}\left\|\mu_{i}\right\|_{p}\left(\Pi_{i}\left(H^{\prime}\right)-\left(\Pi_{i}\left(H^{\prime \prime}\right) \cup \Pi_{i}(H)\right)\right) \leq$

$\leq \sup _{i \geq j, i_{H}} \mid i \mu_{j} \|_{p}\left(\Pi_{j i}^{-1}\left(G_{j}\right)-\Pi_{i}(H)\right) \leq \epsilon$

as stated.

iii) If $\left(H_{j}\right)_{j \epsilon J} \subset \mathscr{E}$ is a decreasing filtering net then

$$
\lambda\left(\bigcap_{j \in J} H_{j}\right)=\lim _{j} \lambda\left(H_{j}\right) .
$$

Let us suppose first that $\bigcap_{j \in J} H_{j}=\emptyset$. In this case $H_{r} \subset \cup_{j \in J}\left(E-H_{j}\right)$ for every $r \in J$; and it follows from $5.1^{j \epsilon J}$ that for every $p \epsilon \mathscr{P}$ and $\epsilon>_{0}^{j \epsilon J} 0$ there exists $k \epsilon J$ and $i_{o} \in I$ such that

$$
|| \mu_{i} \|_{p}\left(\Pi_{i}\left(H_{r}\right)\right) \leq \epsilon
$$

for every $r \geq k(r \in J)$ and $i \geq i_{o}, i_{H_{r}}(i \in I)$. Therefore,

$p\left(\lambda\left(H_{r}\right)\right)=\lim _{i \geq i_{o}, i_{H_{r}}} p\left(\mu_{i}\left(\Pi_{i}\left(H_{r}\right)\right)\right) \leq \sup _{i \geq i_{0}, i_{H_{r}}}|| \mu_{i} \|_{p}\left(\Pi_{i}\left(H_{r}\right)\right) \leq \epsilon$

for every $r \geq k(r \in J)$; hence

$$
\lim _{j} \lambda\left(H_{j}\right)=0=\lambda(\varphi) .
$$


Let us consider the general case. Let $p \epsilon \mathscr{P}$ and $\epsilon>0$. As we have proved before, since $\bigcap_{j \in J} H_{j} \in \mathscr{X}$; there exists $G \in \mathscr{G}$ such that $\bigcap_{j \in J} H_{j} \subset G$ and, if $H^{\prime}$, $H^{\prime \prime} \in \mathscr{\mathscr { E }}$ verify $\bigcap_{j \in J} H_{j} \subset H^{\prime}$ and $H^{\prime}-G \subset H^{\prime \prime} \subset H^{\prime}-\bigcap_{j \in J} H_{j}$, then we get

$$
p\left(\lambda\left(H^{\prime}\right)-\lambda\left(H^{\prime \prime}\right)-\lambda(H)\right) \leq \epsilon / 2 .
$$

Therefore, if for every $j \epsilon J$ we introduce $H_{j}=H_{j}-G$, then we get

$$
p\left(\lambda\left(H_{j}\right)-\lambda\left(H_{j}\right)-\lambda\left(\cap_{r}, H_{r}\right)\right) \leq \epsilon / 2 .
$$

Moreover, since $\bigcap_{\epsilon \in} H_{j}^{\prime}=\emptyset$, there exists $j_{o} \epsilon J$ such that $p\left(\lambda\left(H_{j}\right)\right) \leq \epsilon / 2$ for every $j \geq j_{o}(j \in J)$; and therefore,

$p\left(\lambda\left(H_{j}\right)-\lambda\left(\bigcap_{r \in J} H_{r}\right)\right) \leq p\left(\lambda\left(H_{j}\right)-\lambda\left(\bigcap_{r \in J} H_{r}\right)-\lambda\left(H_{j}^{\prime}\right)\right)+p\left(\lambda\left(H_{j}\right)\right) \leq \epsilon$

for every $j \geq \dot{j}_{0}$; and

$$
\lambda\left(\bigcap_{j \in J} H_{j}\right)=\lim _{j} \lambda\left(H_{j}\right) .
$$

iiv) For every $B \in \mathscr{Q}$, the net $(\lambda(H))_{H \in P(B) \cap y}$ is convergent.

In fact, for every $p \epsilon \mathscr{P}$ and $\epsilon>0$, there exists $\eta>0$ and $i_{o} \epsilon I$ such that $\left\|\mu_{i}\right\|_{p}\left(B_{i}\right) \leq \epsilon / 2$ for every $i \geq i_{o}(i \in I)$ and $B_{i} \in \mathscr{D}_{i}$ with $\nu_{i}^{P}\left(B_{i}\right) \leq \eta$.

Set

$$
\hat{\lambda}_{p}(H)=\inf \left\{\nu_{i}^{p}\left(\Pi_{i}(H)\right): i \geq i_{H}\right\}
$$

for every $H \in \mathscr{H}$; then

$$
r_{B}=\sup \left\{\bar{\lambda}_{\rho}(H): B \supset H \in \mathscr{H}\right\}<+\infty
$$

for everv $B \in \mathscr{B}$, and there exists $H_{o} \in \mathscr{H}_{B}$ (with $\mathscr{H}_{B}=P(B) \cap \mathscr{H}$ ) such that $r_{B}-\eta / 2 \leq \hat{\lambda}_{P}\left(H_{o}\right) \leq r_{B} ;$ and so,

$$
0 \leq \hat{\lambda}_{p}(H)-\hat{\lambda}_{p}\left(H_{o}\right) \leq \eta / 2
$$

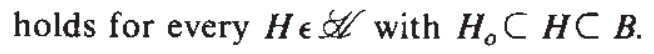

Moreover, there exists $r \in I$ such that $r \geq i_{o}, i_{H}, i_{H_{o}}$ and

$$
\hat{\lambda}_{p}(H) \leq \nu_{j}^{p}\left(\Pi_{j}(H)\right) \leq \hat{\lambda}_{p}(H)+\eta / 2
$$

for all $j \geq r(j \in J)$. Therefore,

$\nu_{j}^{p}\left(\Pi_{j}(H)-\Pi_{j}\left(H_{o}\right)\right)=\nu_{j}^{p}\left(\Pi_{j}(H)\right)-\nu_{j}^{p}\left(\Pi_{j}\left(H_{o}\right)\right) \leq$ 
$\leq \hat{\lambda}_{p}(H)+\eta / 2-\hat{\lambda}_{p}\left(H_{o}\right) \leq \eta$

for all $j \geq r$. Then,

$p\left(\lambda(H)-\lambda\left(H_{o}\right)\right)=\lim _{j \geq r_{,} i_{H}, i_{H_{o}}} p\left(\mu_{j}\left(\Pi_{j}(H)-\Pi_{j}\left(H_{o}\right)\right)\right) \leq$

$\leq \sup _{\nexists r, i_{H}, i_{H_{o}}}\left\|\mu_{j}\right\|_{p}\left(\Pi_{j}(H)-\Pi_{j}\left(H_{o}\right)\right) \leq \epsilon / 2$,

and

$$
p\left(\lambda(H)-\lambda\left(H^{\prime}\right)\right) \leq \epsilon
$$

if $H, H^{\prime} \in \mathscr{U}_{B}, H_{o} \subset H \subset B$ and $H_{o} \subset H^{\prime} \subset B$. The result now follows immediately since the space $X$ is complete.

i) The mapping

$$
\begin{aligned}
\mu: \mathscr{B} & \longrightarrow X \\
B & \longrightarrow \mu(B)=\lim _{H \in \mathscr{X}_{B}} \lambda(H)
\end{aligned}
$$

is well defined, and clearly $\mu(H)=\lambda(H)$ for every $H \epsilon \mathscr{H}$.

vi) For every $B \epsilon \mathscr{B}$, the equality

$$
\mu(B)=\mu(B \cap F)+\mu(B-F)
$$

holds for every closed subset $F \subset E$.

In fact, let $B \in \mathscr{B}, F$ be a closed subset of $E, p \epsilon S D$ and $\epsilon>0$; then there exist $H, H_{1}, H_{2} \in \mathscr{Z}$ such that $H \subset B$,

$H_{1} \subset B \cap F, H_{2} \subset B-F, p(\lambda(K)-\mu(B)) \leq \epsilon / 4$,

$p\left(\lambda\left(K^{\prime}\right)-\mu(B \cap F)\right) \leq \epsilon / 4$ and $p\left(\lambda\left(K^{\prime \prime}\right)-\mu(B-F)\right) \leq \epsilon / 4$ for every $K, K^{\prime}$, $K^{\prime \prime} \epsilon \mathscr{W}$ such that $H \subset K \subset B, H_{1} \subset K^{\prime} \subset B \cap F$ and $H_{2} \subset K^{\prime \prime} \subset B-F$.

Moreover, as we have proved in ii), there exists an open subset $G \subset E$ such that $H_{1} \cup(H \cap F) \subset G$ and

$$
p\left(\lambda\left(H^{\prime}\right)-\lambda\left(H^{\prime \prime}\right)-\lambda\left(H_{1} \cup(H \cap F)\right)\right) \leq \epsilon / 4
$$

if $H^{\prime}, H^{\prime \prime} \in \mathscr{H}$ are such that $H_{1} \cup(H \cap F) \subset H^{\prime}$ and $H^{\prime}-G \subset H^{\prime \prime} \subset H^{\prime}-\left(H_{1} \cup(H \cap F)\right)$. 
Let be $H^{\prime}=H \cup H_{1} \cup H_{2}$ and $H^{\prime \prime}=H_{2} \cup(H-G)$. Then $H^{\prime}, H^{\prime \prime} \in \mathscr{H}$, $H_{1} \cup(H \cap F) \subset H^{\prime}, H^{\prime}-G \subset H^{\prime \prime} \subset H^{\prime}-\left(H_{1} \cup(H \cap F)\right), H \subset H^{\prime} \subset B_{\text {s }}$

$H_{1} \subset H_{1} \cup(H \cap F) \subset B \cap F$ and $H_{2} \subset H^{\prime \prime} \subset B-F$; and therefore,

$p(\mu(B)-\mu(B \cap F)-\mu(B-F)) \leq$

$\leq p\left(\mu(B)-\lambda\left(H^{\prime}\right)\right)+p\left(\lambda\left(H^{\prime}\right)-\lambda\left(H^{\prime \prime}\right)-\lambda\left(H_{1} \cup(H \cap F)\right)\right)+$

$+p\left(\lambda\left(H^{\prime \prime}\right)-\mu(B-F)\right)+p\left(\lambda\left(H_{1} \cup(H \cap F)\right)-\mu(B \cap F)\right) \leq \epsilon$, and [5.2] holds trivially.

vii) If $\left(A_{n}\right)_{n \in N} \subset \mathscr{D}$ is a decreasing sequence then

$$
\mu\left(\cap_{n \in N} A_{n}\right)=\lim _{n \rightarrow+\infty} \mu\left(A_{n}\right) .
$$

Let $p \epsilon \mathscr{P}$ and $\epsilon>0 ;$ then there exists $H_{1} \epsilon \mathscr{H}$ such that $H_{1} \subset A_{1}$ and

$$
p\left(\lambda(H)-\lambda\left(H_{1}\right)\right) \leq \epsilon / 8
$$

if $H \epsilon \mathscr{Y}$ and $H_{1} \subset H \subset A_{1}$. Thus, if $H \epsilon \mathscr{Z}$ verifies $H \subset A_{1}-H_{1}$, we get

$$
p(\lambda(H))=p\left(\lambda\left(H \cup H_{1}\right)-\lambda\left(H_{1}\right)\right) \leq_{\epsilon} / 8 ;
$$

and therefore, $p(\mu(B)) \leq \epsilon / 8$ for every $B \epsilon \mathscr{B}$ with $B \subset A_{1}-H_{1}$.

Moreover, there exists $\mathrm{H}_{2} \in \mathscr{H}$ such that $\mathrm{H}_{2} \subset \mathrm{A}_{2} \cap \mathrm{H}_{1}$ and $p\left(\lambda(H)-\lambda\left(H_{2}\right) \leq \epsilon / 16\right.$ if $H \epsilon \mathbb{C}$ and $H_{2} \subset H \subset A_{2} \cap H_{1}$. Consequently, if $H \in \mathscr{I}$ and $H_{2} \subset H \subset A_{2}$ we have

$p\left(\lambda(H)-\lambda\left(H_{2}\right)\right) \leq p\left(\lambda(H)-\lambda\left(H \cap H_{1}\right)\right)+p\left(\lambda\left(H \cap H_{1}\right)-\lambda\left(H_{2}\right)\right) \leq$

$\leq p\left(\lambda(H)-\lambda\left(H \cap H_{1}\right)\right)+\epsilon / 16=p\left(\mu\left(H-H_{1}\right)\right)+\epsilon / 16 \leq$

$\leq \epsilon / 8(1+1 / 2)$.

So, if $H \in \mathscr{Z}$ and $H \subset A_{2}-H_{2}$ then

$p(\lambda(H))=p\left(\lambda\left(H \cup H_{2}\right)-\lambda\left(H_{2}\right)\right) \leq \epsilon / 8(1+1 / 2)$

and $p(\mu(B)) \leq \epsilon / 8(1+1 / 2)$ for every $B \epsilon \mathscr{B}$ with $B \subset A_{2}-H_{2}$. In particular,

$$
p\left(\mu\left(A_{2}\right)-\lambda\left(H_{2}\right)\right)=p\left(\mu\left(A_{2}-H_{2}\right)\right)<\epsilon / 4 .
$$

Proceeding in this way, we construct a sequence $\left(H_{n}\right)_{n \in N} \subset \mathscr{J}$ such that $H_{n} \subset A_{n} \cap H_{n-1}$ for $n \geq 2$ and 


$$
p(\mu(B)) \leq \epsilon / 8\left(\sum_{\mathrm{j}=0}^{n-1} 1 / 2^{j}\right) \quad(<\epsilon / 4)
$$

for every $B \in$ such that $B \subset A_{n}-H_{n}$ (and in particular,

$\left.p\left(\mu\left(A_{n}\right)-\lambda\left(H_{n}\right)\right)=p\left(\mu\left(A_{n}-H_{n}\right)\right)<\epsilon / 4\right) \quad\left(n \in N^{*}\right)$.

Since $\left(H_{n}\right)_{n \in N}$ is a decreasing sequence, we get

$$
\lambda\left(\cap_{n \in N} H_{n}\right)=\lim _{n \rightarrow+\infty} \lambda\left(H_{n}\right) .
$$

and there exists $n_{o} \epsilon N$ such that

$$
p\left(\lambda\left(H_{m}\right)-\lambda\left(\bigcap_{n \in N} H_{n}\right)\right) \leq \epsilon / 6
$$

for every $m \geq n_{0}$.

Moreover, there exists $H \in \mathscr{H}$ such that $\bigcap_{n \in N} H_{n} \subset H \subset \bigcap_{n \in N} A_{n}$ and

$$
p\left(\lambda(H)-\mu\left(\cap_{n \in N} A_{n}\right) \leq \in / 6 ;\right.
$$

the sequence ( $\left.H \cap H_{n}\right)_{n \in N} \subset \mathscr{H}$ is decreasing, and there exists $n_{1} \in N$ such that

$$
p\left(\lambda\left(H \cap H_{m}\right)-\lambda\left(\cap_{n \in N} H_{n}\right)\right) \leq \epsilon / 6
$$

for all $n \geq n_{1}$.

So, if $m \geq \max \left(n_{0}, n_{1}\right)$, then

$p\left(\mu\left(A_{m}\right)-\mu\left(\bigcap_{n \in N} A_{n}\right)\right) \leq$

$\leq p\left(\mu\left(A_{m}\right)-\lambda\left(H_{m}\right)\right)+p\left(\lambda\left(H_{m}\right)-\lambda\left(\bigcap_{n \in N} H_{n}\right)\right)+$

$+p\left(\lambda\left(\cap_{n \in N} H_{n}\right)-\lambda\left(H \cap H_{m}\right)\right)+p\left(\lambda\left(H \cap H_{m}\right)-\lambda(H)\right)+$

$+p\left(\lambda(H)-\mu\left(\bigcap_{n \in N} A_{n}\right)\right)<\epsilon$;

and [5.3] holds.

viii) As it is easily proved, the set

$\mathscr{P}=\{A \in \mathscr{B}: \mu(B)=\mu(B \cap A)+\mu(B-A)$ for all $B \in \mathscr{B}\}$

is an algebra. 
ix) If $\left(A_{n}\right)_{n \in N} \subset \mathscr{L}$ is an increasing sequence, then $\cup_{n \in N} A_{n} \in \mathscr{L}$ and

$$
\left.\mu\left(B \cap \cup_{n \in N} A_{n}\right)\right)=\lim _{n \rightarrow+\infty} \mu\left(B \cap A_{n}\right)
$$

holds for every $B \epsilon \mathscr{B}$.

In fact, if $\left(A_{n}\right)_{n \in N} \subset \mathscr{L}$ is an increasing sequence and $B \in \mathscr{B}$,

then $\cap_{m \in N}\left(\left(B \cap\left(\cup_{n \in N} A_{n}\right)\right)-A_{m}\right)=\emptyset$ and

$\left.0=\lim _{m} \mu\left(B \cap\left(\cup_{n \in N} A_{n}\right)\right)-A_{m}\right)=$

$=\mu\left(B \cap\left(\bigcup_{n \in N} A_{n}\right)\right)-\lim _{m} \mu\left(B \cap A_{m}\right) ;$

and therefore,

$\mu\left(B \cap\left(\cup_{n \in N} A_{n}\right)\right)+\mu\left(B-\cup_{n \in N} A_{n}\right)=$

$=\lim _{m} \mu\left(B \cap A_{m}\right)+\lim _{m} \mu\left(B-A_{m}\right)=\mu(B)$,

and $\bigcup_{n \in N} A_{n} \in \mathscr{L}$.

x) Evidently, $\mu$ is a finitely additive vector measure and $\mathscr{L}$ is a $\sigma$-algebra.

xi) $\mu$ is a Radon measure of type ( $\mathscr{\&})$.

Let $A \in \mathscr{B}, p \in \mathscr{P}$ and $\epsilon>0$; then there exists $H \epsilon \mathscr{Z}$ such that $H \subset A$ and

$$
p(\lambda(F)-\lambda(H)) \leq \epsilon / 2
$$

if $F \in \mathscr{Z}$ is such that $H \subset F \subset A$. Thus, if $F \in \mathscr{\&}$ is such that $F \subset A-H$ then

$$
p(\lambda(F))=p(\lambda(F \cup H)-\lambda(H)) \leq \epsilon / 2,
$$

and $p(\mu(B)) \leq \epsilon / 2$ for every $B \in \mathscr{B}$ with $B \subset A-H$. Therefore,

$\|\mu\|_{p}(A-H) \leq 2 \sup \{p(\mu(B)): B \epsilon \mathscr{B}, B \subset A-H\} \leq \epsilon$.

Moreover, every $H \epsilon \dot{H}$ is $\mu$-compact, since for every open cover $\left\{G_{j}\right\}_{j \epsilon \zeta}$ of $H, p \in \mathscr{P}$ and $\epsilon>0$, we get from condition 5.1 the existence of a finite subset $J^{\prime} \subset J$ and $i_{o} \in I$ with $i_{o} \geq i_{H_{1}}$, with $H_{1}=H-\bigcup_{j \in J^{\prime}} G_{j}$, such that

$\left\|\mu_{i}\right\|_{p}\left(\Pi_{i}\left(H-\bigcup_{j \in J^{\prime}} G_{j}\right)\right) \leq \epsilon / 2$ for every $i \geq i_{o}$.

So, if $H^{\prime} \epsilon \mathscr{H}$ and $H^{\prime} \subset H-\bigcup_{j \in J^{\prime}} G_{j}$, then 
$p\left(\mu\left(H^{\prime}\right)\right)=\lim _{i \geq_{v^{\prime}, H_{H}}} p\left(\mu_{i}\left(\Pi_{i}\left(H^{\prime}\right)\right)\right) \leq \sup _{i i_{\sigma^{*}} i_{H^{*}}}\left\|\mu_{i}\right\|_{p}\left(\Pi_{i}\left(H^{\prime}\right)\right) \leq \epsilon / 2 ;$

and

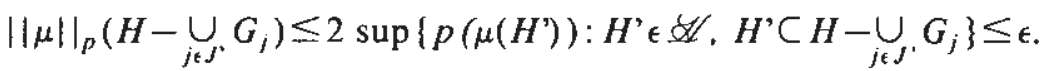

xii) It follows from the last results that $\mu$ is $\sigma$-additive.

х̇ii)

$$
\mu\left(\Pi_{i}^{-1}\left(H_{i}\right)\right)=\mu_{i}(/ /)
$$

for every $i \epsilon I$ and $H_{i} \epsilon \mathscr{H}_{i}$.

In fact, it follows from the condition 5.2 that for every $i \in I, H_{i} \in \mathscr{Z}_{i}, p \in \mathscr{P}$ and $\epsilon>0$, there exists $H \epsilon \not{K}$ such that $H \subset \Pi_{i}^{-1}\left(H_{i}\right)$ and

$$
\left\|\mu_{j}\right\|_{p}\left(\Pi_{i j}^{-1}\left(H_{j}\right)-\Pi_{j}(H)\right) \leq \epsilon / 2
$$

for every $j \geq i, i_{H}$. Moreover, there exists $H^{\prime} \epsilon \mathscr{Z}$ such that $H \subset H^{\prime} \subset \mathrm{II}_{i}^{-1}\left(H_{i}\right)$ and

$$
p\left(\mu\left(\Pi_{i}^{-1}\left(H_{i}\right)\right)-\lambda\left(H^{\prime}\right)\right) \leq \epsilon / 2 .
$$

Therefore,

$p\left(\mu\left(\mathrm{II}_{i}^{-1}\left(H_{i}\right)\right)-\mu_{i}\left(H_{i}\right)\right) \leq$

$\leq p\left(\mu\left(\Pi_{i}^{-1}\left(H_{i}\right)\right)-\lambda\left(H^{\prime}\right)\right)+p\left(\lambda\left(H^{*}\right)-\mu_{i}\left(H_{i}\right)\right) \leq$

$\leq_{\epsilon} / 2+\lim _{j \geq i_{i}, i_{H}} p\left(\mu_{j}\left(\Pi_{j}\left(H^{\prime}\right)\right)-\mu_{i}\left(H_{j}\right)\right)=$

$=\epsilon / 2+\lim _{j \geq i_{,} i_{H}, i_{H}} p\left(\mu_{j}\left(\mathrm{II}_{j}\left(H^{\prime}\right)\right)-\mu_{j}\left(\mathrm{II}_{i j}^{-1}\left(H_{j}\right)\right)\right) \leq$

$\leq \epsilon / 2+\sup _{j \geq i_{i} i_{H}, i_{H^{\prime}}}\left\|\mu_{j}\right\|_{p}\left(\mathrm{II}_{i j}^{-1}\left(H_{i}\right)-\mathrm{II}_{j}\left(H^{\prime}\right)\right) \leq$

$\leq \epsilon / 2+\sup _{j \geq i, i_{H}}\left\|\mu_{j}\right\|_{p}\left(\Pi_{i j}^{-1}\left(H_{i}\right)-\Pi_{j}(H)\right) \leq \epsilon ;$

and $\mu_{i}\left(H_{j}\right)=\mu\left(\Pi_{i}^{-1}\left(H_{i}\right)\right)$.

xivi) Let us prove that $\mu$ is the projective limit of $\left(\mu_{i}\right)_{i \in}$.

For this, we consider the family

$$
\mathscr{P}_{i}=\left\{A_{i} \in \mathscr{O}_{i}: \mu_{i}\left(A_{i}\right)=\mu\left(\Pi_{i}^{-1}\left(A_{i}\right)\right)\right\} \quad(i \in I) .
$$


Then, if $F_{i}$ is a closed subset of $E_{i}$, then for every $p \epsilon \mathscr{P}$ and $\epsilon>0$, there exists $H_{i} \epsilon \mathscr{K}_{i}$ and $H \epsilon \mathscr{C}$ such that $H_{i} \subset F_{i}, H \subset \Pi_{i}^{-1}\left(F_{i}\right),\left\|\mu_{i}\right\|_{p}\left(F_{i}-H_{i}\right) \leq \epsilon / 2$ and $\|\mu\|_{p}\left(\Pi_{i}^{-1}\left(F_{i}\right)-H\right) \leq \epsilon / 2$. Therefore,

$p\left(\mu_{i}\left(F_{i}\right)-\mu\left(\Pi_{i}^{-1}\left(F_{i}\right)\right)\right) \leq$

$\leq p\left(\mu_{i}\left(F_{i}\right)-\mu_{i}\left(H_{i} \cup \overline{\Pi_{i}(H)}\right)\right)+p\left(\mu_{i}\left(H_{i} \cup \overline{\Pi_{i}(H)}\right)-\mu\left(\Pi_{i}^{-1}\left(F_{i}\right)\right)\right) \leq$

$\leq|| \mu_{i}\left\|_{p}\left(F_{i}-\left(H_{i} \cup \overline{\Pi_{i}(H)}\right)\right)+\right\| \mu \|_{p}\left(\Pi_{i}^{-1}\left(F_{i}\right)-H\right) \leq \epsilon ;$

and $F_{i} \in \mathscr{S}_{i}$.

Moreover, if $A_{i}, B_{i} \in \mathscr{S}_{i}$ and $A_{i} \subset B_{i}$ then $B_{i}-A_{i} \in \mathscr{S}_{i}$; and $\bigcup_{n \in N} A_{n}^{i} \in \mathscr{S}_{i}$ for every increasing sequence $\left(A_{n}^{i}\right)_{n \in N} \subset \mathscr{S}_{i}$.

Now it follows immediatley that $\mathscr{S}_{i}=\mathscr{D}_{i}$, and that $\mu_{i}\left(A_{i}\right)=\mu\left(\Pi_{i}^{-1}\left(A_{i}\right)\right)$ for every $i \in I$ and $A_{i} \in \mathscr{B}_{i}$, as we wanted to prove.

This ends the proof of Theorem 5.

Let us consider now a Hausdorff and complete locally convex space $X$ which is the projective limit of a projective system $\left(X_{i}, f_{i}\right)_{i, j \in I}$ of Hausdorff and complete locally convex spaces and denote by $\mathscr{P}_{i}$ a generating and saturated family of seminorms on $X_{i}$ (we will assume that if $i, j \in I, p_{i} \in \mathscr{P}_{i}$ and $i \leq j$, then $\left.p_{i} f_{i j} \in \mathscr{P}_{j}\right)$, and by $f_{i}: X \longrightarrow X_{i}(i \in l)$ the canonical projection.

As we have made before, let $\left(\left(E_{i}, \mathscr{G}_{i}\right), \Pi_{i j}\right)_{i, j \in I}$ be a projective system of topological spaces, with $\mathscr{B}_{i}$ the Borel $\sigma$-algebra of $E_{i}, \mathscr{H}_{i}$ a family of closed subsets of $E_{j}$, which is closed under finite unions, and $\mu_{i}: \mathscr{B}_{i} \longrightarrow X_{i}$ a Radon measure of type $\left(\mathscr{X}_{i}\right)$.

Let $E=\lim E_{i}$ and denote by $\mathscr{B}$ the Borel $\sigma$-algebra of $E$ and by $\Pi_{i}: E \rightarrow E_{i}(i \in I)$ the canonical projection.

We will assume that

$$
\mu_{i}\left(A_{j}\right)=f_{i j}\left(\mu_{j}\left(\Pi_{i j}^{-1}\left(A_{j}\right)\right)\right)
$$

for every $A_{i} \in \mathscr{B}_{i}, i, j \in I$ with $i \leq j$.

Definition 6. We say that a measure $\mu: \mathscr{B} \longrightarrow X$ is the projective limit (it is easily proved that if the projective limit measure exists then it is unique) of the last system of measures $\left(\mu_{i}\right)_{i \in}$ if

$$
f_{i}\left(\mu\left(\Pi_{i}^{-1}\left(A_{i}\right)\right)\right)=\mu_{i}\left(A_{i}\right)
$$

for every $A_{i} \in \mathscr{B}_{i}$ and $i \in I$. 
Let us assume that $\mathscr{E}$ is a class of closed subsets of $E$ which verifies the condition 3.1, and that for every $i \epsilon I$ and every $p_{i} \epsilon \mathscr{S}_{i}{ }^{\prime}$ re exists a non negative and finite measure

$\nu_{i}^{P_{i}}: \mathscr{B}_{i} \rightarrow \mathbb{R}^{+}$such that:

a) The equality

$\nu_{i}^{p_{i}}\left(A_{i}\right)=\inf \left\{\nu_{i}^{p_{i}}(G): A_{i} \subset G, G\right.$ open subset of $\left.E_{i}\right\}$ holds for every $i \in I$, $p_{i} \in \mathscr{P}_{i}$ and $A_{i} \in \mathscr{S}_{i}$.

b) For every $i \epsilon I, p_{i} \in \mathscr{P}_{i}$ and $\epsilon>0$ there exists $i_{0} \geq i$ and $\eta>0$ such that if $j \in I$ and $A_{j} \in \mathscr{B}_{j}$ are such that $i_{0} \leq j$ and $\nu_{j}^{p_{i} f_{i j}}\left(A_{j}\right) \leq \eta$, then

$$
|| \mu_{j}||_{p_{i} f_{i j}}\left(A_{j}\right)<\epsilon .
$$

c) $\Pi_{i j}\left(v_{j}^{p_{i} f_{i j}}\right)=\nu_{i}^{p_{i}}$ holds for every $i, j \in I$ with $i \leq j$ and every seminorm $p_{i} \in S^{-1}$

Then proceeding like in last proofs, the following results are obtained:

Proposition 7. For every $H \in \mathscr{H}$ and $i \in I,\left(f_{i j}\left(\mu_{j} \Pi_{j}(H)\right)\right)_{j \geq i_{H}}$ is a convergent net in $X_{i}$; and the mapping $\lambda: \mathscr{H} \longrightarrow X$ such that

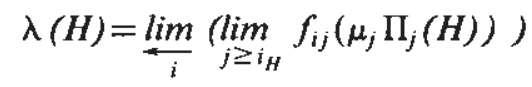

is well defined.

Theorem 8. The projective limit $\mu$ of the (last) projective system of measures $\left(\mu_{i}\right)_{i \epsilon}$ exits and $\mu: \mathscr{B} \longrightarrow X$ is a Radon measure of type (XX), if and only if the following conditions are fulfilled:

8.1. For every $H \epsilon \mathscr{H}, i \in I, p_{i} \epsilon \mathscr{P}_{i}, \epsilon>0$ and every open cover $\left\{G_{r}\right\}_{r \in L}$ of $H$, there exists a finite subset $L^{\prime} \subset L$ and $i_{o} \in I$ such that $i_{o} \geq i$, $i_{H_{1}}$, with $H_{1}=H-\bigcup_{r \in L}, G_{r}$, and

$$
|| \mu_{j}||_{p_{i} f_{i j}}\left(\Pi_{j}\left(H-\bigcup_{r \in L}, G_{r}\right)\right) \leq \epsilon
$$

holds for every $j \geq i_{o}$.

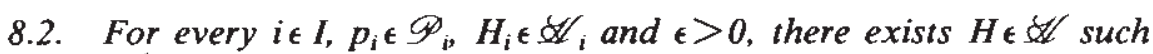
that $H \subset \Pi_{i}^{-1}\left(H_{j}\right)$ and

$$
|| \mu_{j} \|_{p_{i}} f_{i j}\left(\Pi_{i j}^{-1}\left(H_{i}\right)-\Pi_{j}(H)\right) \leq \epsilon
$$

for every $j \geq i, i_{H}$. 
Moreover, if $\mu=\lim \mu_{i}$ exists and is a Radon measure of type ( $\mathscr{O}$ ), then it is unique and $\mu(\overline{H)}=\lambda(H)$ for every $H \epsilon \& f, \lambda$ being the set function defined in Proposition 7. Also, the measure $\mu$ is of bounded semivariation if and only if the semivariations $\left\{|| \mu_{j} \|_{p_{i} f_{i j}}: j \geq i\right\}$ are uniformly bounded for every $i \epsilon I$ and every $p_{i} \in \mathscr{P}_{i}$.

\section{ON THE PROJECTIVE LIMIT OF PRODUCT MEASURES}

Let us continue with the notations of last section and consider two projective systems of Hausdorff and complete locally convex spaces

$\left(Y_{i}, g_{i j}\right)_{i, j \in I}$ and $\left(Z_{i}, h_{i j}\right)_{i, j \in f}$, with $Q_{i}$ (resp., $\left.\mathscr{R}_{i}\right)$ a generating (and saturated) family of seminorms on $Y_{i}$ (resp. on $\left.Z_{i}\right)(i \in I)$, and suppose that $q_{i} g_{i j} \in Q_{j}$ and $r_{i} h_{i j} \in \mathscr{R}_{j}$ for every pair of seminorms $q_{i} \in Q_{i}, r_{i} \in \mathscr{R}_{i}$ and every $i, j \in I$ with $i \leq j$. We shall write $Y=\lim Y_{i}, Z=\lim Z_{i}, \mathscr{D}=\left\{p_{i} f_{i}: p_{i} \in \mathscr{D}_{i}, i \in I\right\}, Q=\left\{q_{i} g_{i}\right.$ :

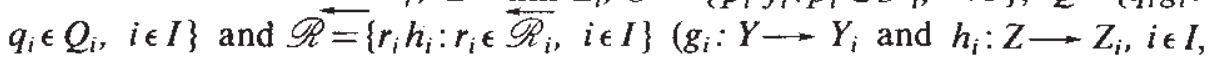
will be the natural projections as usual).

Let us consider another projective system of Radon measures of type $\left(\mathscr{F F}_{i}\right),\left(\left(F_{i}, \mathscr{G}_{i}\right), \Pi_{i j}, \Pi_{i}, \nu_{i}\right)_{i, j \in l} l$, where $\mathscr{F}_{i}$ is a family of closed subsets of $F_{i}$, closed under finite unions, $\mathscr{D}_{i}^{\prime}$ is the Borel $\sigma$-algebra of $F_{i}$ and $\nu_{i}: \mathscr{B}_{i}{ }^{\prime} \rightarrow Y_{i}$ is a Radon measure of type $\left(\mathscr{F}_{i}\right), i \in I$ (see definition 3 ).

Let $\mathscr{B}$ ' be the Borel $\sigma$-algebra of $F$, where $F=\lim F_{i}$; we shall use the following notation: $\Pi_{i}: F \longrightarrow F_{i}$ is the natural projection ( $i \in I$ ), $\hat{\Pi}_{i j}=\left(\Pi_{i j}, \Pi_{i j}\right), l_{i j}=\left(f_{i j}, g_{i j}\right), \hat{\Pi}_{i}=\left(\Pi_{i}, \Pi_{i}^{\prime}\right)$ and $l_{i}=\left(f_{i}, g_{i}\right)$ for all $i, j \in I$ with $i \leq j$.

Suppose that, for every $i \in I$, there exists a bilinear and continuous mapping $\delta_{i}: X_{i} \times Y_{i}-Z_{i}$ such that the following diagram is commutative for $i \leq j(i, j \in I)$ :

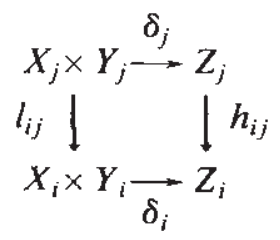

and let $\delta: X \times Y \rightarrow Z$ be the function $\left(\delta_{i}\right)_{i \epsilon}$.

Theorem 9. If the projective limits $\mu$ and $\nu$ of the systems of measures $\left(\mu_{i}\right)_{i \in I}$ and $\left(\nu_{i}\right)_{i \in I}\left(\mu=\lim \mu_{i}, \nu=\lim \nu_{i}\right)$ and the product measures $\mu_{i} \otimes \nu_{i}(i \in I)$ 
exist, then the product measure $\mu \otimes \nu$ exists if and only if the projective limit measure (lim $\left.\mu_{i} \otimes \nu_{i}\right)$ of the system of measures $\left(\mu_{i} \otimes \nu_{i}\right)_{i \in}$ exists, and in this case they coincide.

Proof. Let us suppose that the measure $\gamma=\lim \mu_{i} \otimes \nu_{i}$ exists; then it is easily proved that for every $A_{i} \in S_{i}$ and $i \in I$ the equality

$$
\gamma\left(\mathrm{II}_{i}^{-1}\left(A_{i}\right) \times B\right)=\delta\left(\mu\left(\Pi_{i}^{-1}\left(A_{i}\right)\right), \nu(B)\right)
$$

holds for every $B \in \mathscr{B}$, from where it is deduced that

$$
\gamma(A \times B)=\delta(\mu(A), \nu(B))
$$

is verified for every $A \epsilon \notin$ and every $B \in B$ ' and consequently $\gamma=\mu \otimes \nu$.

The other implication is trivial.

\section{References}

[1] BELI.EY, J. and MORALES, P.: Régularité d'une fonction d'ensembles à valeurs dans un groupe topologique. Ann. sc. Québec (1979).

[2] Chorksi, J. R.: Inverse limits of measure spaces. Proc. London Math. Soc., 8 (1958), 321-342.

[3] Diestel, J. and UHL, J. J.: Vector measures. Math. Surveys (15). Amer. Math. Soc. Providence, 1977.

[4] Dobrakov, I.: Products of operator valued measures and the Fubini theorem. Abstracts of the Fourth Prague Topological Symposium, 1976.

[5] DucHoN, M.: On the projective tensor product of vector-valued measures, I. Mat. Cas. 17 (1967), 113-120.

[6] -: On the projective tensor product of vector-valued measures, II. Mat. Cas. 19 (1969), 228-234.

[7] -: On tensor product of vector measures in locally compact spaces. Mat. C̆as. 19 (1969), 324-329.

[8] -: On vector measures in Cartesian products. Mat. Cas.21 (1971), 241-247.

[9] - : Fubini's theorem and convolution of vector valued measures. Mat. Cas. 23 (1973), 170-178.

[10] --: Product of dominated vector measures. Math. Slovaca 27 (1977), 293-301.

[11] DuchoÑ, M. and KLuvanek, I.: Inductive tensor product of vector-valued measures. Mat. Cas. 17 (1967), 108-112.

[12] Fernandez, J. and Jimenez Guerra, P.: Opérateurs dans les spaces de mesures. Ann. Sc. Math. Québec, 7 (1983), 95-101.

[13] Fernández Fernández-Arroyo, F. J.: Producto de medidas valoradas en espacios localmente convexos. Ph. D. Thesis, U.N.E.D., Madrid, 1987.

[14] - : Sobre la existencia del producto de medidas valoradas en espacios localmente convexos: Una condición necesaria. Pub. Mat., 32 (1988), 129-134. 
[15] - : On the product of operators valued measures. (To appear).

[16] Jiménez Guerra, P. and Rodriguez-Salinas, B.: Medidas de Radón de tipo (U) en espacios topológicos arbitrarios. Mem. R. Acad. Ci. Madrid, t. 10, 1979.

[17] KLUVÁNEK, I.: An example concerning the projective tensor product of vectorvalued measures. Mat. Cas. 20 (1970), 81-83.

[18] Ki.unánek, I. and KNOWlis, G.: Vector Measures and Control Systems. North-Holland. Amsterdam (1975).

[19] MARĹA GONZÁLEZ, J. L.: \&Z/-regularidad para medidas vectoriales. Rev. R. Acad. Ci. Madrid, 76 (1982), 681-686.

[20] MÉtrier, M.: Limites projectives de mesures, martingales et applications. Annali di Mat., 63 (1963), 225-352.

[21] MORALES, P.: Regularity and extensions of semigroup-valued measures. Lect. Notes in Math., 794 (1979).

[22] Musial, K.: Inverse limits of measure spaces. Bull. Acad. Polon. Sci. Sér. Sci. Math. Astronom. Phys., 19 (1971), 437-440.

[23] PROKHOROV, U. V.: Convergence of random processes and limit theorems in probability theory. Theor. Prob: Appl., 1 (1956), 157-214.

[24] Rao Chivukula, R. and SASTRY, A. S.: Product vector meastures via Bartle integrals. J. Math. Anal. Appl., 96 (1983), 180-195.

[25] STORY, D.: A Fubini theorem for inductive tensor product-measures. Rev. Roum. Math., 24 (1979), 161-165.

[26] SCHWARTZ, L.: Radon measures on arbitrary topological spaces and cylindrical measures. Oxford Univ. Press, 1973.

[27] SWARTZ, C.: The product of vector-valued measures. Bull. Australian Math. Soc., 8 (1973), 359-366.

[28] —: Products of vector meastures. Mat. Cas. 24 (1974), 289-299.

[29] -: A generalization of a theorem of Duchon on products of vector measures. J. Math. Anal. Appl. 51 (1975), 621-628.

[30] SwarTz, C.: Products of vector measures by means of Fubini's theorem. Math. Slovaca 27 (1977), 375-382.

[31] _-: Fubini's theorem for tensor product measures. Rev. Roumaine Math. Pures Appl. 29 (1984), 97-103.

[32] VAlkova, T. V.: Projective limits of measure spaces of their automorphisms. Uspehi Mat. Nauk 25 (1970), n.o 6 (156), 219-220.

[33] Vasilach, S.: Direct limils of measure spaces. J. Multivariante Anal., I (1971), 394-411.

Departamento de Matemáticas Fundamentales Facultad de Ciencias - UNED

c/ Senda del Rey, s/n-Ciudad Universitaria 28040 Madric: 